\title{
Spectrum Sensing Experimentation for LTE and WiFi Unlicensed Band Operation
}

\author{
Nenad Milošević, Zorica Nikolić, Filip Jelenković, Valentina Nejković, and Milorad Tošić
}

\begin{abstract}
If several different systems operate in the same frequency band, a coordination between them is needed for effective use of the available spectrum. The coordination is especially important if the systems are not designed to operate in such an environment. The very important initial phase of the coordination process is acquiring of the spectrum usage map or spectrum sensing. The paper describes the spectrum sensing experimentation in the unlicensed $5 \mathrm{GHz}$ band during the WiFi or LTE transmission. It describes the experiment workflow and depicts the obtained results. The experiments were performed at NITOS testbed at the University of Thessaly, Greece, and show that it is possible to determine whether WiFi or LTE transmission is sensed. Therefore, based on spectrum sensing it will be possible to coordinate a shared access of WiFi and LTE users in the unlicensed $5 \mathrm{GHz}$ band.

Keywords - Cognitive radio, spectrum sensing, unlicensed band, LTE, WiFi.
\end{abstract}

\section{INTRODUCTION}

A S already known, the amount of data transferred over mobile data networks is rapidly increasing. The predictions say that the mobile data traffic will increase many times in the following years [1].

The mobile communications high data rates, high capacity and excellent quality of service were mainly fulfilled with the introduction of long-term evolution (LTE) standard that was first defined in 3GPP Release 8

Paper received July 12, 2016; revised September 12, 2016; accepted September 14, 2016. Date of publication November 20, 2016. The associate editor coordinating the review of this manuscript and approving it for publication was Prof. Ljiljana Milić.

This paper is a revised and expanded version of the paper presented at the 23rd Telecommunications Forum TELFOR 2015 [12].

The research leading to these results has received funding in part from the European Union's Seventh Framework Programme under grant agreement no 612050 (FLEX Project) and in part by the Ministry of Science and Technological Development of Serbia within the Project TR32037.

Nenad Milošević is with the Faculty of Electronic Engineering, University of Niš, Aleksandra Medvedeva 14, 18000 Niš, Serbia (phone: 381-18-529367; e-mail: nemilose@elfak.ni.ac.rs).

Zorica Nikolić is with the Faculty of Electronic Engineering, University of Niš, Aleksandra Medvedeva 14, 18000 Niš, Serbia (phone: 381-18-529245; e-mail: zora@elfak.ni.ac.rs).

Filip Jelenković is with the Faculty of Electronic Engineering, University of Niš, Aleksandra Medvedeva 14, 18000 Niš, Serbia (phone: 381-18-529323; e-mail: filipjelenkovic@gmail.com).

Valentina Nejković is with the Faculty of Electronic Engineering, University of Niš, Aleksandra Medvedeva 14, 18000 Niš, Serbia (phone: 381-18-529524; e-mail: valentina.nejkovic@elfak.ni.ac.rs).

Milorad Tošić is with the Faculty of Electronic Engineering, University of Niš, Aleksandra Medvedeva 14, 18000 Niš, Serbia (phone: 381-18-529323; e-mail: mbtosic@yahoo.com).
[2]. However, LTE will not be able to support high data rates in the following years. One relatively simple way to increase the data rate is to use a higher spectrum bandwidth. Since the licensed bands, assigned to the mobile communication systems, are mostly occupied, some of the unlicensed bands may be used for the bandwidth extension. Because of this, among other reasons, the LTE standard was further improved in the 3GPP Release 10 [3]. It was named LTE-Advanced, or LTE-A, and is aimed at meeting the requirements of $4 \mathrm{G}$ mobile networks. Simultaneous use of multiple frequency bands, named Carrier Aggregation (CA), is the most important improvement of the LTE-A. CA is the key technology that enables the unlicensed spectrum usage by the LTE devices.

Any wireless communication system may freely use the unlicensed band, but in order to ensure efficient coexistence between different systems operating in the unlicensed spectrum, some regulations or coordination mechanisms have to be followed. All stations, operating in an unlicensed band, are required to employ some mechanism that takes care of other users and fair spectrum sharing. The mechanism is the dynamic spectrum access (DSA), and it may have different variants. Some of them are listen-before-talking (LBT) and dynamic frequency selection (DFS). The frequency selection in DSA systems is based on the measured data regarding the spectrum occupancy or on the spectrum sensing. Spectrum sensing is used to detect occupied and unoccupied parts of the considered spectrum and provide data for the process of spectrum usage coordination. Different SS techniques are adopted [4], [5] including traditional detect-and-avoid approach, spectrum cartography, and cooperative spectrum sensing.

This paper describes the spectrum sensing experimentation in the unlicensed band, which is studied within the CoordSS (Coordination by Spectrum Sensing for LTE-U) project, being a part of the FLEX (FIRE LTE testbeds for open experimentation) project [6]. The spectrum sensing is an important part of the experimentation framework that would support LTE-U self-organizing networks exploiting unlicensed spectrum at $5 \mathrm{GHz}$ for the extension of the licensed band using the carrier aggregation technology.

The rest of the paper is organized as follows. Section II explains the most important technologies in LTEAdvanced and gives some facts regarding the unlicensed bands. Section III describes the experiment, and the concluding remarks are given in Section IV. 


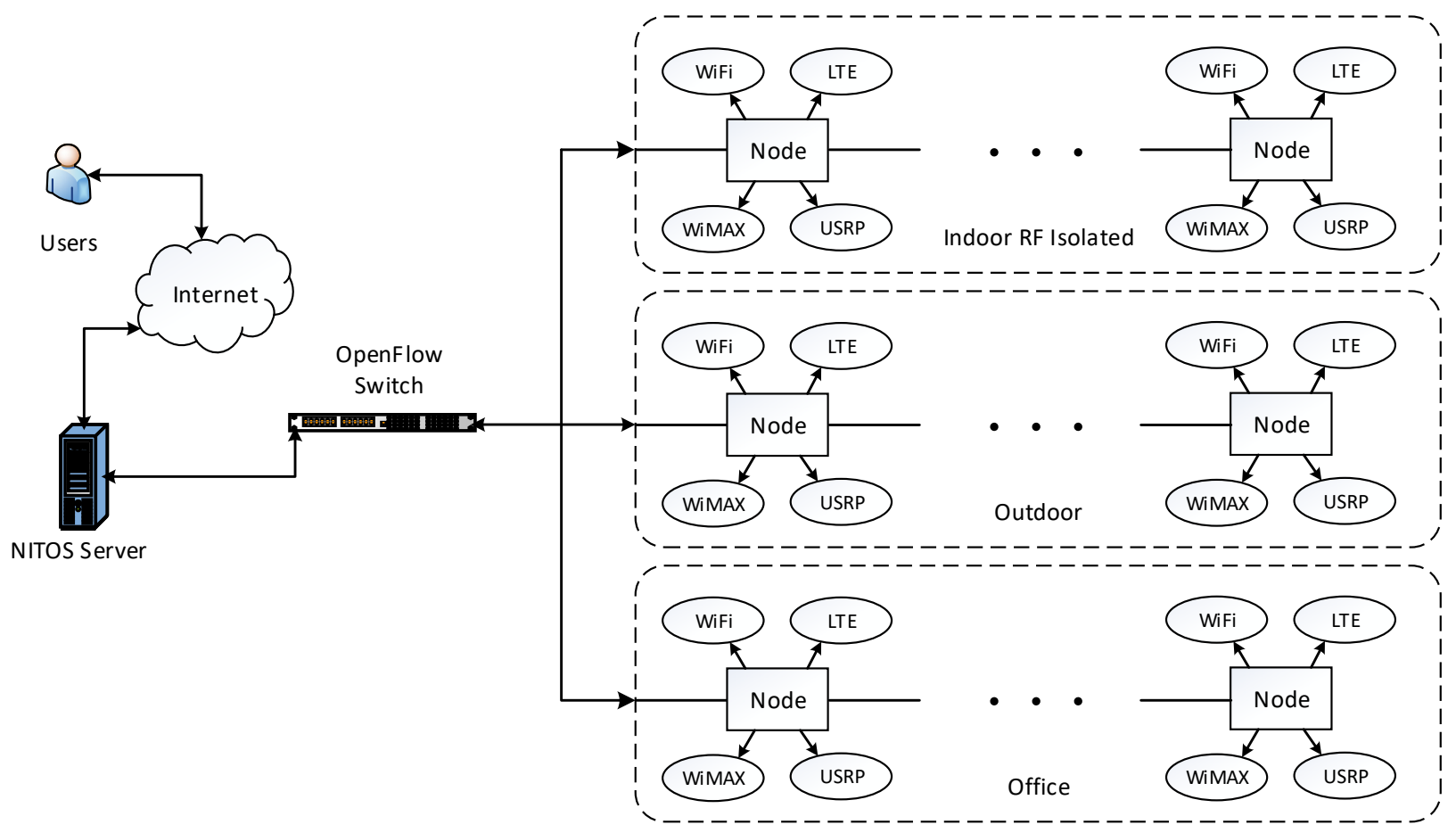

Fig. 1 NITOS testbed block diagram [8].

\section{LONG TERM EVOLUTION - ADVANCED AND THE UNLICENSED BANDS}

LTE-Advanced, as stated, is an improved version of LTE, introduced in 3GPP Release 10. Carrier aggregation is the most important new feature of LTE-A, regarding the use of the unlicensed spectrum. CA allows LTE to simultaneously use multiple carriers, even at different frequency bands, and therefore, because of a higher bandwidth, to achieve a higher bitrate. At the same time, LTE-A is backward compatible with previous releases (8 and 9) of the LTE. Release-8 LTE supports carrier bandwidths of $1.4,3,5,10,15$, and $20 \mathrm{MHz}$. LTE-A may use up to five Release- 8 carriers. Therefore, a LTE-A terminal may use up to $5 \times 20=100 \mathrm{MHz}$ of bandwidth.

The unlicensed band (UB) consists of a number of sub bands, from several $\mathrm{MHz}$, up to several hundreds of $\mathrm{GHz}$. UB mainly consists of ISM (Industrial, Scientific and Medical) and U-NII (Unlicensed National Information Infrastructure) bands. ISM bands were not primarily intended to be used for communication purposes, but due to the rapid development of communication technology, the ISM bands are used by communications systems, such as Bluetooth, near field communications (NFC), WiFi and other. ISM frequency bands, being of interest for communication purposes, are 902-928 MHz, 2400-2500 $\mathrm{MHz}$, and 5.725-5.875 GHz. U-NII bands cover frequencies from $5.125-5.925 \mathrm{GHz}$, where 5.725-5.850 $\mathrm{GHz}$ U-NII sub band overlap with ISM band. LTE operation in unlicensed band has the potential to offer significantly better coverage and higher spectral efficiency compared to $\mathrm{WiFi}$, while allowing seamless flow of data across licensed and unlicensed in a single core network [7].It is planned to use $5-5.8 \mathrm{GHz}$ band in the beginning. Also, at first downlink unlicensed communications will be developed, and later the uplink traffic will also be extended to the unlicensed band. The unlicensed spectrum would only be used for data rate increase, both in downlink and uplink. However, the licensed spectrum, having a predictable performance, will still be used for the important operations, and for the delivery of a guaranteed quality of service.

When using the unlicensed spectrum, a fair coexistence of LTE and other technologies, such as WiFi, has to be ensured. Upon the unlicensed spectrum usage, LTE network observes the spectrum, selects the channel with the least interference, and dynamically adjusts for the continued interference avoidance. The channel status is usually acquired by spectrum sensing. Spectrum sensing may be performed by a single device that needs to communicate or, preferably, by all network devices. They measure signal levels in different frequency bands and report the results to the network controller that analyzes the measurements and makes the decision about the spectrum that is going to be used. Both of these processes, spectrum sensing and spectrum management or coordination, are equally important. In this paper we describe the spectrum sensing methodology in an experiment, and the spectrum coordination will be the subject of the future work.

\section{EXPERIMENT: LTE-U/WIFI SPECTRUM SENSING}

The experiment was executed at the NITOS testbed, located at the University of Thessaly, Volos, Greece, and accessible via Internet [8]. The testbed consists of three parts: Outdoor testbed, Indoor Office testbed, and Indoor $\mathrm{RF}$ Isolated testbed. The general block diagram of the testbed is shown in Fig. 1. Indoor RF Isolated testbed was used for the experiment, because of its RF isolation and therefore controlled environment. The topology of this 
testbed is shown in Fig. 2. It consists of 44 nodes arranged in a grid (11 rows with 4 nodes each) with $1 \mathrm{~m}$ distance between neighboring nodes. Nodes are equipped with different wireless and wired communication devices, such as WiFi, WiMax, and LTE. Also, some nodes are equipped with USRP (Universal Software Radio Peripheral) B210 devices, and one of these nodes was used for spectrum sensing. Besides, a pair of USRP nodes served as LTE eNB and UE (User Equipment). Since there is no dedicated commercial LTE equipment operating in the $5 \mathrm{GHz}$ unlicensed band, a software radio implementation of the LTE standard, named OpenAirInterface [9] was used. It should be noted that the nodes are not mobile and therefore the hadrware configuration of the nodes cannot be changed online, i.e. only people with the physical access to the testbed are able to change the hardware configuration of the nodes.

Spectrum sensing was performed by the Wideband Software Extensible Radio Platform (WiSER) [10], developed at OrbitLab [11]. WiSER uses USRP for spectrum sensing and signal generating. WiSER is implemented through the wiserd software. wiserd receives parameters via a command line, configures USRP for the needed task, receives data from USRP and writes results to the database.

The spectrum sensing node is at USRP equipped node56, and the $\mathrm{WiFi}$ nodes are 50 and 68. The OpenAirInterface is located at nodes 59 and 60. The spectrum is sensed in the unlicensed $5 \mathrm{GHz}$ band, using frequency $5.24 \mathrm{GHz}$, which is WiFi channel 48. Spectrum sensing parameters are summarized in the following Table.

TABLE 1: SPECTRUM SENSING PARAMETERS.

\begin{tabular}{|l|l|}
\hline Parameter & Value \\
\hline Central frequency & $5.24 \mathrm{GHz}$ \\
\hline FFT length & 512 \\
\hline Sensing time & $2000 \mathrm{~ms}$ \\
\hline Averaging window size & 500 \\
\hline
\end{tabular}

In the first part of the experiment, the spectrum is sensed with WiFi stations on and off, and with LTE stations off. The experiment consists of the following steps:

- Nodes imaging

Prior to imaging, the nodes have to be turned off.:

omf tell -a off -t node050, node056, node068

Now, the nodes 50, 56, and 68are imaged with wiserdnitos.ndz image:

omf load -I wiserd-nitos.ndz -t

node050, node056, node068

After imaging, the imaged nodes have to be turned on:

omf tell -a on -t node050, node056, node068

- Experiment execution

The experiment is executed with:

omf exec wiserd-sensing.rb--slice username wiserd-sensing.rb is the file with the experiment description source code.

\section{- Results collecting}

The results are at the sensing node 56 within the file /root/sensed_spectrum_node56.txt. We need to ssh to 56 copy results to local computer.

- Results visualisation

The visualised results are shown in Fig.3.

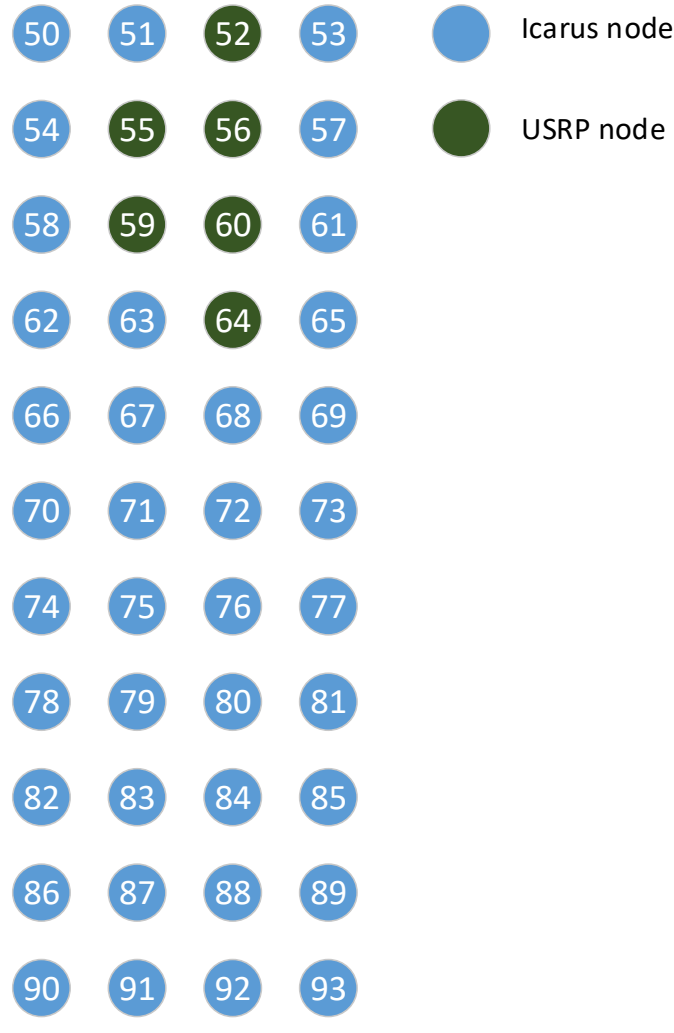

Fig. 2 Indoor RF Isolated testbed topology [8].

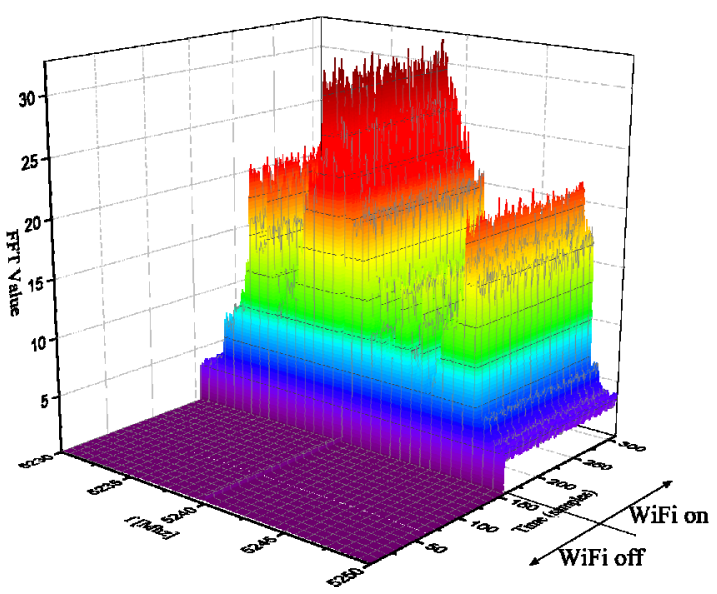

Fig.3 Sensed spectrum with and without WiFi.

The WiFi is initially turned off, up to about sample 150 . After that, from sample 150 to sample 300, the WiFi is turned on. As can be seen from Fig.3, WiFi activity may be identified, because the USRP senses only noise from sample 0 to sample 150 , and there is much higher sensed spectrum power from samples 150 to 300 . 
The experiment is described with the Ruby code, i.e. the contents of wiserd-sensing.rb file is:

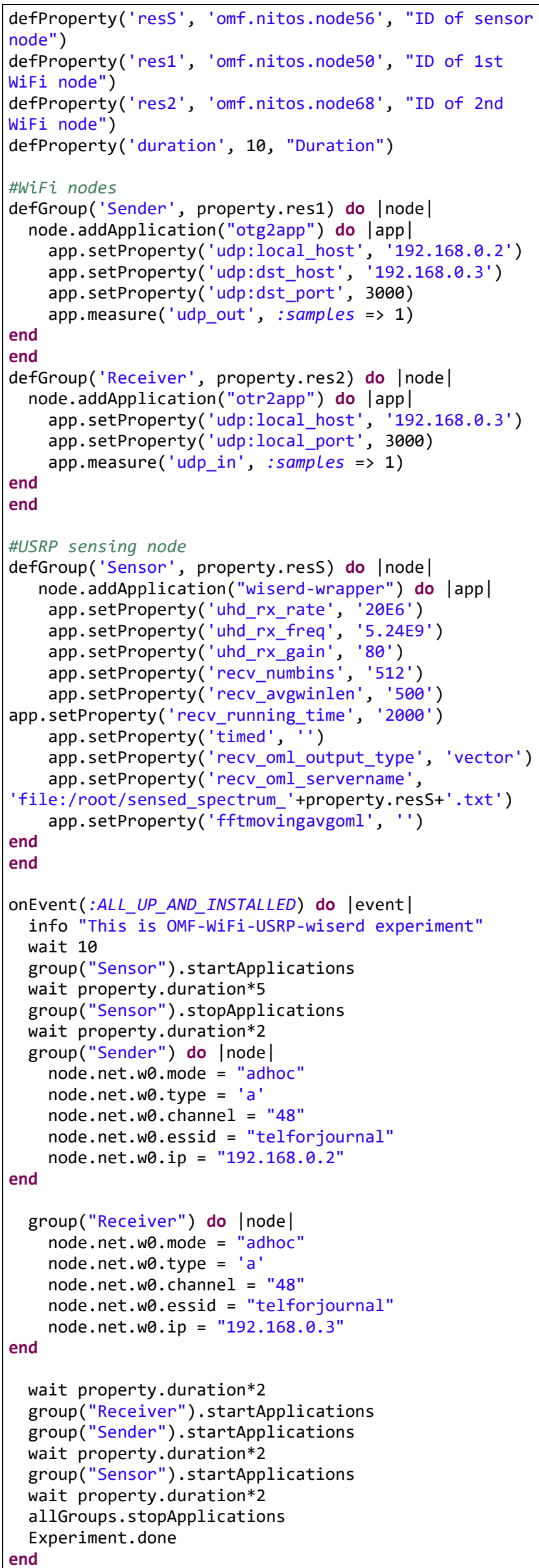

During the second part of the experiment, the spectrum was sensed with the LTE stations turned on. Some of the LTE parameters are summarized in Table 2.

TABLE 2: LTE PARAMETERS.

\begin{tabular}{|l|l|}
\hline Parameter & Value \\
\hline Downlink frequency & $5.24 \mathrm{GHz}$ \\
\hline Uplink frequency & $5.14 \mathrm{GHz}$ \\
\hline Duplexing mode & $\begin{array}{l}\text { FDD (Frequency } \\
\text { Division Duplex) }\end{array}$ \\
\hline $\begin{array}{l}\text { Number of resource } \\
\text { blocks } N_{R B}\end{array}$ & 25 \\
\hline Channel bandwidth & $5 \mathrm{MHz}$ \\
\hline
\end{tabular}

Two cases are considered: 1) eNB is turned on and UE is off, where eNB transmits only control packets, and 2) $\mathrm{UE}$ is attached to eNB and some downlink traffic is generated between them.

Figs. 4 and 5 show the visualized spectrum for these two cases.

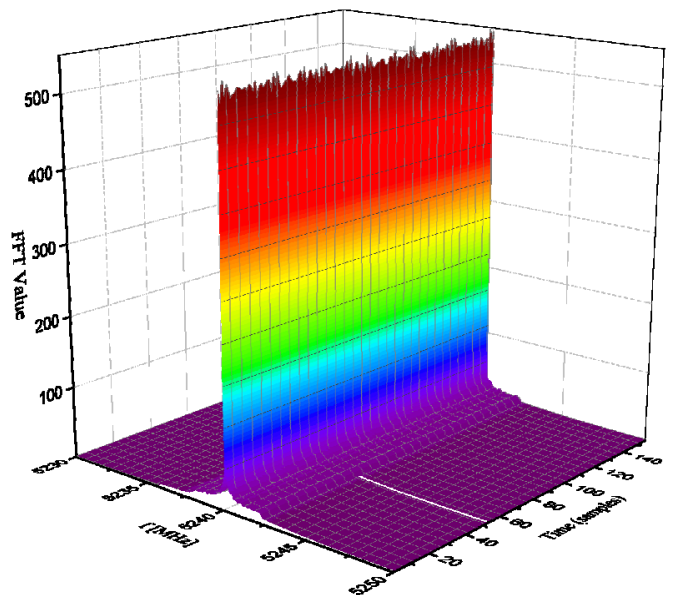

Fig. 4 Sensed spectrum with eNB only.

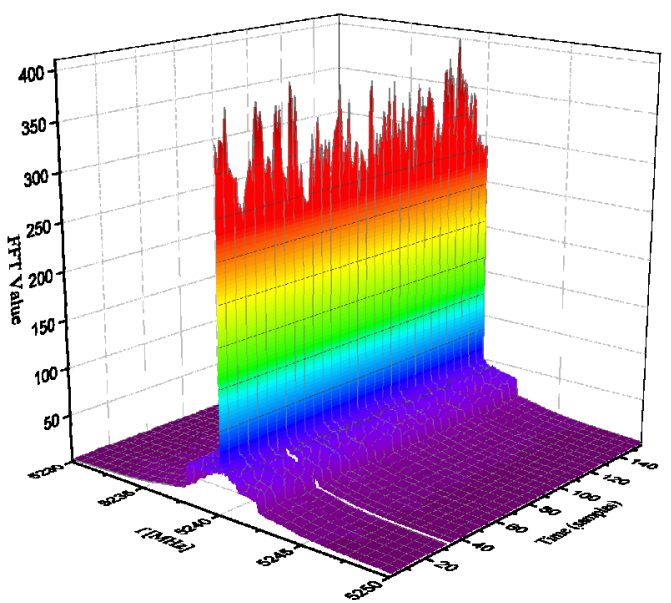

Fig. 5 Sensed spectrum with eNB + UE.

The time-average values of the sensed spectra are shown in Fig. 6. 


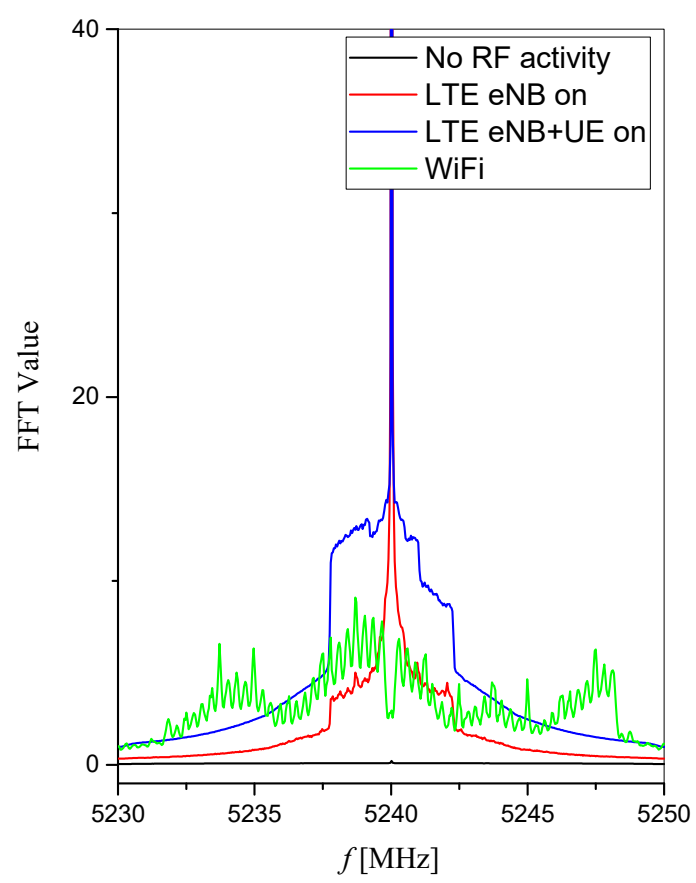

Fig. 6 Sensed spectra averaged over time.

Sensed spectra show the difference between WiFi and LTE. LTE has a strong peak at the downlink central frequency, so it is possible to distinguish what kind of signal is present.

\section{CONCLUSION}

This paper described the spectrum sensing experimentation at NITOS testbed within the NITLab at the University of Thessaly, Greece. The spectrum sensing is one of the most important processes needed for the dynamic spectrum access and spectrum coordination. The experiments have shown the WiFi and LTE activity may be identified and therefore coordinated with other systems operating in the unlicensed band.
The next step is to develop a system that would be able, based on measured data, to coordinate a shared access of LTE and WiFi devices operating in the unlicensed $5 \mathrm{GHz}$ band, in order to achieve an optimal performance with minimum interference between different users.

\section{ACKNOWLEDGMENT}

The authors thank the anonymous reviewers for their valuable suggestions and comments.

\section{REFERENCES}

[1] Qualcomm (2013), "Extending the benefits of LTE Advanced to unlicensed spectrum", [Online] Available: http://www.qualcomm. $\mathrm{com} / \mathrm{media} /$ documents/files/extending-the-benefits-of-lte-advancedto-unlicensed-spectrum.pdf

[2] 3GPP (2015), “3GPP Release 8," [Online] Available: http://www.3gpp.org/specifications/releases/72-release-8

[3] 3GPP (2015), “3GPP Release 10," [Online] Available: http://www.3gpp.org/specifications/releases/70-release-10

[4] S. Haykin, D. J. Thomson, and J. H. Reed, "Spectrum sensing for cognitive radio," Proceedings of the IEEE, vol. 97, no. 5, pp. 849877, May, 2009.

[5] D. D Ariananda, M. K. Lakshmanan, H. Nikookar, "A survey on spectrum sensing techniques for cognitive radio," in Proc. CogART 2009, Aalborg, Danmark , pp.74-79, 18-20 May 2009

[6] FLEX (FIRE LTE testbeds for open experimentation), [Online] Available: http://www.flex-project.eu/

[7] Qualcomm Research, "LTE in Unlicensed Spectrum: Harmonious Coexistence with Wi-Fi," White Paper, accessed 9. Oct. 2014, [Online] Available: https://www.qualcomm.com/media/documents /files/lte-unlicensed-coexistence-whitepaper.pdf

[8] Network Implementation Testbed using Open Source (NITOS), [Online] Avail.: http://nitlab.inf.uth.gr/NITlab/index.php/nitos.html

[9] OpenAirInterface Software Alliance, OpenAirInterface, accessed 18. May 2016, [Online] Available: http://www.openairinterface.org/

[10] OrbitLAB, Wideband Software Extensible Radio Platform (WiSER), [Online]. Available: http://wiser.orbit-lab.org/wiki/wiser

[11] OrbitLAB, [Online]. Available: http://www.orbit-lab.org

[12] N. Milošević, Z. Nikolić, F. Jelenković, V. Nejkovič, M. Tošić and I. Šeškar, "Spectrum sensing for the unlicensed band cognitive radio," Telecommunications Forum Telfor (TELFOR), 2015 23rd, Belgrade, 2015, pp. 250-252. 